\title{
Estimación de daños y pérdidas por desastres de origen natural durante el año 2010 para los departamentos de Francisco Morazán y El Paraíso, Honduras
}

\author{
Óscar Elvir, Skarleth Motiño, Pablo Domínguez
}

\section{RESUMEN}

Este trabajo propone dar continuidad al desarrollo de una base de datos en materia de daños y pérdidas por desastres de origen natural para Honduras, no obstante, se seleccionó específicamente la zona de estudio en los departamentos de Francisco Morazán y El Paraíso, para el año 2010 y, a partir de esto, se realizó una estimación monetaria del impacto causado por los desastres provocados por amenazas de origen natural en los sectores más vulnerables, tales como los sociales (vivienda, salud), infraestructura (vial, energía, acueductos y alcantarillados) y económicos (agricultura, ganadería).

Posteriormente, se espera agregar dicha información al sistema DesInventar (Inventario de Desastres) de Honduras; con lo cual se busca poner a prueba una metodología para valorar la estimación de daños y pérdidas por desastres y así poder contar con información que permita guiar los planes de rehabilitación y reconstrucción, con la finalidad de promover la prevención y reducción del riesgo de desastres.

Palabras clave: desastre, natural, inundación, sequía, incendio, Copeco, deslizamiento, tormenta, tropical, huracán, pérdida, daño, producción, economía, educación, social, vivienda, infraestructura vial, Cepal, estimación, riesgo, amenaza, mitigación.

\section{ABSTRACT}

The study pretends to continue the development of a database in terms of damages and losses that result from natural disasters in Honduras, specifically selecting the departments of Francisco Morazán and El Paraíso as study areas, for the year 2010. This will estimate the monetary impact that is caused by natural hazards in vulnerable sectors such as the social sector (housing, health), infrastructure (roads,

\footnotetext{
${ }^{1}$ Universidad Nacional Autónoma de Honduras. Facultad de Ciencias, Instituto Hondureño de Ciencias de la Tierra. Correo electrónico: elvirferman@yahoo.com.mx; pdominguez17@gmail.com; www.ichit.edu.hn.
} 
power, water and sewage) and the economic sector (agriculture, livestock). The information will be added to the disaster inventory system of Honduras, named DesInventar, in order to test a methodology that can assess the damage and estimate disaster losses. This information will guide rehabilitation and reconstruction plans in order to promote prevention and reduce disaster risk.

Keywords: disaster, natural, flood, drought, fire, Copeco, landslides, storm, tropical, hurricane, loss, damage, production, economy, education, social housing, road infrastructure, Cepal, estimation, risk, hazard, mitigation.

\section{INTRODUCCIÓN}

Los desastres de origen natural surgen de la interacción y coincidencia en un tiempo y espacio dado, de un fenómeno natural potencialmente destructivo (amenaza) y condiciones de vulnerabilidad dentro de las comunidades y entornos en los cuales impacta el fenómeno. Para ejemplificar mejor este concepto se suele expresar que el desastre es la conjugación de amenazas y vulnerabilidad, con lo cual ambos factores se constituyen en condicionantes para que se exista riesgo de un desastre y su posterior consumación, si no es bien manejado.

Honduras es el segundo país más grande y montañoso de América Central, con una extensión territorial de 112, $492 \mathrm{~km}^{2}$ y una población aproximada de 7,7 millones de habitantes. Su posición geográfica hace que los huracanes afecten el país todos los años, ocasionando inundaciones principalmente en la zona norte (costa Caribe) del país. Paralelamente, la intensidad de las precipitaciones tropicales durante la estación lluviosa acentúan el impacto de las inundaciones, afectando la salud, producción agrícola, el sector vivienda, la infraestructura vial y, principalmente, a la población que habita en zonas de alto riesgo.

Pero, las inundaciones no son el único evento que pone en riesgo al país, también lo afectan los deslizamientos, incendios forestales, sequías incrementadas por la presencia del fenómeno El Niño y, en menor medida, la afectación por terremotos, por lo que el país está empezando a adoptar las acciones de prevención, mitigación y atención a emergencias y desastres para preservar la vida y los bienes de la población.

En Honduras existe un gran déficit en materia de capacidad de respuesta a los desastres de origen natural, el gobierno central y organizaciones extranjeras invierten grandes cantidades de fondos para poder fortalecerla; sin embargo, cuando ocurre un desastre, existe solo respuesta a la emergencia, no hay aún un 
enfoque bien desarrollado sobre prevención y mitigación, lo cual es una preocupación constante, ya que los fenómenos naturales ocurren de manera permanente en el transcurso del año en la región centroamericana, atacando a todo el territorio del país, esencialmente en los puntos de mayor vulnerabilidad.

Todos los años ocurren en el país desastres que impactan la economía de las zonas que afectan, provocando daños y pérdidas en sectores como agricultura, vivienda, infraestructura vial, entre otros. Así, en 2010, se presentaron varios eventos que causaron daños y pérdidas en varios municipios del país, entre ellos los más afectados fueron los departamentos de Francisco Morazán y El Paraíso.

Estos daños y pérdidas no han sido valorizados considerando un tipo de metodología que permita estimarlos de una manera integral, para brindar información básica acerca de los impactos de los desastres a los tomadores de decisiones, siendo este uno de los objetivos del presente trabajo que busca aplicar una metodología para realizar tal valoración.

La estimación de daños y pérdidas en las zonas de estudio puede a ayudar a responder preguntas como las siguientes: ¿Cuál es el valor estimado de las pérdidas por desastres de origen natural ocurridas durante el año 2010? ¿Qué sectores han sido los más afectados? ¿Se contribuyó a la mejora del inventario de desastres en el país? ¿De qué forma el análisis de daños y pérdidas por desastres a nivel municipal puede contribuir al fortalecimiento de las capacidades de respuesta a desastres y a la reducción de la vulnerabilidad de los municipios ante las amenazas a las que están expuestos?.

En consecuencia a esta problemática, es de suma importancia contar con metodologías que traten de hacer estimaciones monetarias del impacto generado posterior a un desastre, para que ayuden a contribuir a la reducción del riesgo de desastres en el país.

\section{MÉTODO}

\section{Selección delárea de estudio}

Se determinó como área de estudio a los departamentos de Francisco Morazán y El Paraíso, considerando que durante el año 2010 se registró en estos un significativo impacto debido a desastres de origen natural provocados por eventos como las tormentas tropicales Agatha y Matthew (ver mapa 1). 
Mapa 1. Ubicación general de la zona de estudio

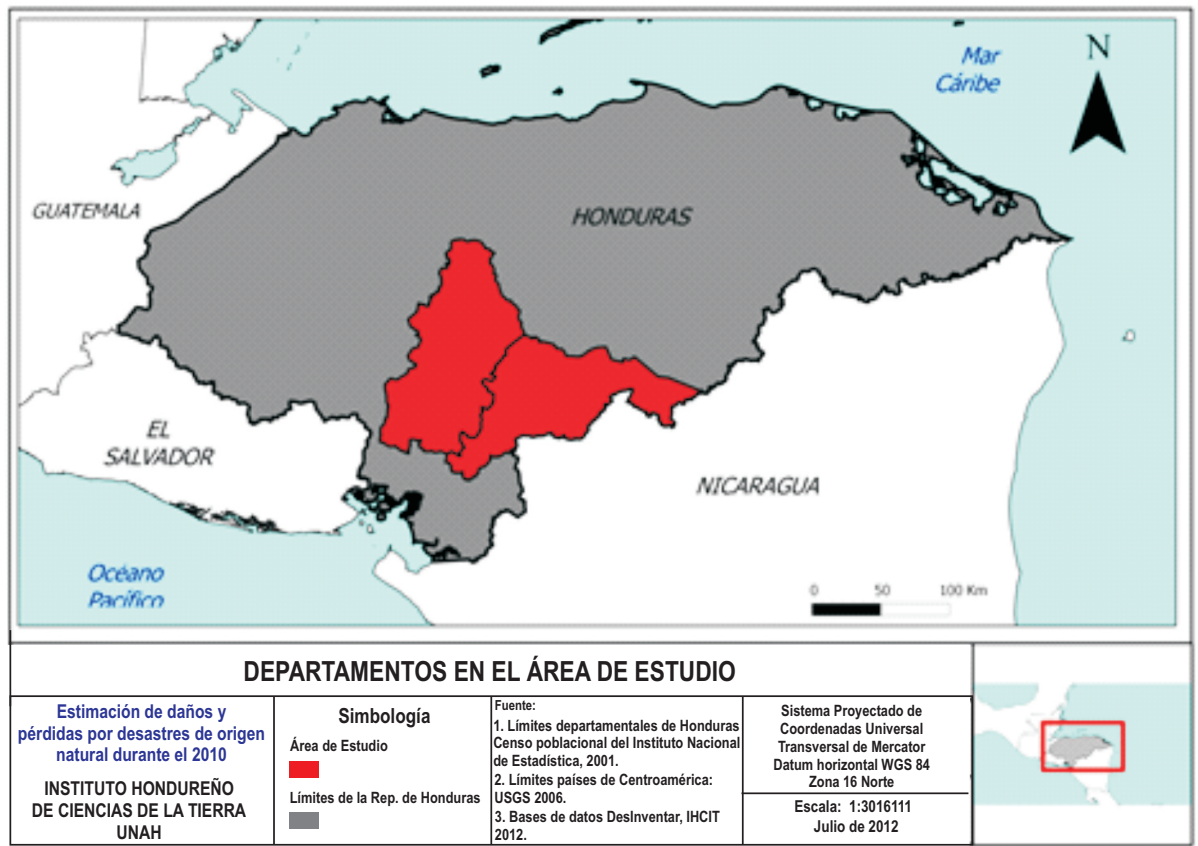

Extracción de datos de Francisco Morazán y El Paraíso desde la base de datos DesInventar

Se realizaron consultas en la base de datos para Honduras del sistema DesInventar, filtrando las fichas correspondientes a los departamentos de Francisco Morazán y El Paraíso. Dichos datos de cada ficha fueron exportados a formato de Excel para poder ser procesados en un editor de hojas de cálculo. Asimismo, se obtuvo información de acerca de daños y pérdidas proveniente de diferentes instituciones del país.

Metodología para la estimación de daños y pérdidas por desastres de origen natural

Metodología seleccionada

Para este estudio se tomó como guía la metodología de evaluación del impacto socioeconómico y ambiental de desastres de la Comisión Económica para América Latina (CEPAL), la cual clasifica las áreas de impacto de un desastre en 3 sectores, que son sector infraestructura, sector económico y sector social. Se tomó como guía la metodología de la CEPAL porque se basa en más de 3 décadas de 
investigación llevada a cabo durante visitas a los lugares donde han ocurrido los desastres, en momentos posteriores a los mismos, siendo capaces de evaluar cuáles son los efectos a corto y largo plazo. En la estimación de daños se recomienda que una vez pasada la etapa de emergencia posterior a un desastre, se haga la evaluación de los efectos directos e indirectos del evento, así como de sus consecuencias para el bienestar social y desarrollo económico.

Los efectos de un fenómeno natural se han clasificado por la CEPAL en daños directos e indirectos. Los directos son aquellos que alteran los acervos, es decir, son los que sufren los activos inmovilizados, destruidos o dañados y los infligidos a las existencias (de bienes finales o en proceso, materias primas, materiales y repuestas). Entre los principales rubros de esta categoría se cuentan la destrucción total o parcial de infraestructura física, edificios, instalaciones, maquinaria, equipos, medios de transporte y almacenaje, mobiliario; perjuicios en las tierras de cultivo, en obras de riego, embalses, etc. En cuanto a la agricultura, la destrucción de la producción que ya estaba lista para ser cosechada debe valorarse e incluirse también como un daño directo.

Este tipo de daños directos por lo general se registran en el momento del desastre o pocas horas después del mismo; generalmente son de duración corta, pero en el caso de los desastres con un largo periodo de gestación como una sequía o el fenómeno El Niño, los efectos también pueden verse durante un largo periodo de tiempo.

Los daños indirectos son los que se producen sobre los flujos de producción de bienes y servicios. Se refieren básicamente a los bienes y servicios que se dejan de producir o de prestar durante un lapso que se inicia después de acaecido el desastre y que puede prolongarse durante la fase de rehabilitación y reconstrucción, la cual convencionalmente se ha establecido en un máximo de cinco años, aunque las mayores pérdidas ocurren durante los dos primeros. En todo caso, el cálculo de su efecto debe extenderse el tiempo que sea requerido para alcanzar la recuperación parcial o total de la capacidad productiva. Por lo general son más difíciles de observar o cuantificar que los daños directos.

El análisis de los daños directos e indirectos se hace por sectores, considerando la siguiente clasificación:

1. Sectores sociales: población afectada, vivienda y asentamientos humanos, educación y cultura, salud.

2. Infraestructura: energía, agua potable y saneamiento, transporte y 
comunicaciones.

3. Sectores económicos: agropecuario, industria y comercio, turismo.

4. Efectos globales de los daños: medioambiente.

Análisis de los factores utilizados para la estimación

Para estimar los daños directos e indirectos (pérdidas) ocasionados por desastres de origen natural ocurridos en el 2010, enfocándose en cada sector, se consideraron los siguientes factores:

1. Sector social: vivienda y asentamientos humanos. La estimación monetaria de daños ocasionados por desastres de origen natural se realizó con base al valor de reposición, considerando los precios actuales de los materiales de construcción, mano de obra o insumos necesarios para reponer, reparar 0 reconstruir las estructuras dañadas o destruidas tomando en cuenta el grado 0 nivel de daño de la vivienda, el tipo de vivienda y la zona en la cual se encontraba ubicada.

2. Sector social: salud. El principal impacto en sector salud durante el año 2010, fue la rápida propagación de la epidemia del dengue, por lo cual se consideró el número de atenciones en hospitales a pacientes con la infección viral del dengue clásico y dengue hemorrágico, así como los costos que implica para el centro hospitalario la atención de los pacientes. También se consideraron los gastos que implica la campaña de prevención de la epidemia del dengue, tomando como principal factor el costo de la compra y distribución de abate en los barrios y colonias del país.

3. Sector infraestructura: transporte y comunicaciones (red vial). Se revisaron exhaustivamente los costos provenientes de materiales de construcción y costos de mano de obra actualizados hasta julio de 2010 en la zona de Tegucigalpa y zona sur, en materia de construcción de pavimentos rígidos con concreto hidráulico, para lo cual se utilizó el manual de precios de la Cámara Hondureña de la Industria de la Construcción (CHICO).

4. Sector infraestructura: distribución de agua potable y saneamiento. Para las estimaciones de daños fue necesario considerar los costos por reparación de cada tramo de acueducto dañado, los cuales son administrados y ejecutados por el Servicio Autónomo Nacional de Acueductos y Alcantarillados (SANAA).

5. Sector infraestructura: energía. El principal recuento de daños en materia de 
energía corresponde a pérdidas por falta de energía eléctrica y daños en circuitos madre e igualmente por el tendido eléctrico dañado por ramas de árboles que detuvieron el flujo de corriente. Por tanto, se solicitó a la ENEE la información correspondiente por reparaciones de estos daños, con la comisión de transparencia de la misma institución, en formato físico y digital para su posterior procesamiento y análisis.

6. Económico: agricultura y ganadería. En este sector se consideraron los daños en agricultura y ganadería (cabezas de ganado vacuno y porcino, cultivos de maicillo, plátano, palma africana, caña de azúcar, frijol, arroz, papa y sorgo, entre otros), se solicitaron los datos a la Secretaría de Agricultura y Ganadería (SAG) para el departamento de Francisco Morazán y El Paraíso (ya que en el municipio del Distrito Central no se registraron daños o pérdidas) correspondientes a los desastres de origen natural con mayor trascendencia, como la tormenta tropical Agatha y las tormentas tropicales de los meses de agosto y septiembre de 2010.

7. Otros sectores: medioambiente. Se debe considerar el impacto económico generado por los daños al medioambiente, sobre todo los causados por incendios forestales que dañan los bosques, ya que estos pueden implicar daños a la industria de la exportación de madera, además del impacto sobre el ecosistema debido a la contaminación de fuentes de agua y emanación de $\mathrm{CO}_{2}$ en la atmósfera.

La metodología de estimación de los daños directos por incendios forestales ha sido desarrollada por el Instituto Hondureño de Conservación y Desarrollo Forestal y Vida Silvestre (ICF). De esta manera, el daño sobre los bosques ocasionados por incendios forestales, se estimó considerando el costo de sustitución de un árbol de pino quemado, además de las actividades de prevención de incendios que involucra:

1. Costo por plantalista para plantación.

2. Costo necesario para establecer apropiadamente una plántula en el sitio definitivo.

3. Costo de construcción por kilómetro de ronda corta fuego con una dimensión de 5 metros de ancho, limpiando con machete y rastrillo-azadón 1 metro en cada lado de la ronda y quemado el centro.

4. Las quemas a realizar, estas se prescriben principalmente para la protección de bosques jóvenes, en regeneración natural, plantaciones establecidas, así como otras que se consideren de importancia. 
5. Protección contra incendios de toda el área bajo manejo.

6. Evaluación y manejo de regeneración natural y plantaciones (podas, limpiezas y aclareos).

Por lo tanto, deben considerarse cada uno de los costos por hectárea de bosque y relacionándolo con el número de hectáreas dañadas, se obtendrá el valor de daños en este sector.

\section{Fuentes y recopilación de información}

1. Sector social: vivienda y asentamientos humanos. Se recolectó información proveniente de la Cámara Hondureña de la Construcción (boletín estadístico) 2010. Asimismo, se revisaron los costos de los materiales de construcción y costos de mano de obra actualizados hasta julio de 2010 en la zona de Tegucigalpa y zona sur, para la posterior estimación de precios en el cuadro de costo de viviendas.

2. Sector social: salud. Se consideraron los costos de atención de un paciente con dengue clásico y de un paciente con dengue hemorrágico; estos datos fueron provistos por la administración de los hospitales públicos, específicamente por el director del Hospital Escuela, Dr. Marco Molinero, y los que fueron publicados por Diario El Heraldo, en su edición del 1 de julio de 2010. También se consideraron como costos indirectos aquellos referentes a la campaña de prevención y los costos de distribución de abate en los barrios y colonias de las zonas de estudio.

3. Sector infraestructura: transporte y comunicaciones (red vial). Se realizaron visitas a la Secretaría de Obras Públicas, Transporte y Vivienda (SOPTRAVI); Alcaldía Municipal del Distrito Central (Catastro), con el objetivo de recopilar información sobre los tramos dañados $(\mathrm{km})$ a causa de desastres de origen natural durante el año 2010. Se enviaron las solicitudes requeridas de los sitios antes mencionados, en físico y digital, mediante las direcciones de correos electrónicos otorgados por la Comisión Permanente de contingencias (COPECO).

4. Sector infraestructura: distribución de agua potable y saneamiento. Se solicitó a la oficina de transparencia del SANAA, por medio de la Lic. Marcela Girón, el monto invertido en reparación de tramos de acueductos y alcantarillados dañados como efecto de los desastres de origen natural, incluyendo una breve 
descripción de los daños, las colonias afectadas y las fechas de dichas reparaciones.

5. Sector infraestructura: energía. Se trabajó directamente con la información provista por la ENEE correspondiente a reparaciones hechas al sistema de suministro de energía eléctrica en las zonas bajo estudio, dicha información esta detallada por zonas (barrios o colonias).

6. Económico: agricultura y ganadería. Se solicitó a la Secretaría de Agricultura y Ganadería, SAG, a través de la oficina de transparencia de dicha institución, datos sobre cantidad de hectáreas de cultivos afectados y cabezas de ganado perdidos durante el año 2010 y el costo directo que implicaron dichos daños.

7. Otros sectores: medioambiente: Los datos fueron brindados por el Instituto Hondureño de Conservación y Desarrollo Forestal, Áreas Protegidas y Vida Silvestre, a través del Ing. Alejandro Somoza.

Proceso de la información recopilada

A. Sector social: vivienda y asentamientos humanos.

Se realizó una categorización de las viviendas y se calculó el costo promedio de cada tipo de vivienda, para así tomar en cuenta el boletín informativo de la Cámara Hondureña de la Construcción 2010 (CHICO) sobre precios de materiales de construcción y mano de obra.

\section{Categorización de las viviendas}

Se clasificó el tipo de vivienda en vivienda rural y vivienda urbana; a su vez, cada una se categorizó de acuerdo a ubicación y área de construcción invertida.

a. Zona urbana: Para este cálculo se consideró un área de 14×25 y materiales estándar.

1) Categoría U1 (urbana-alta):

- Casa de habitación ubicada en una zona residencial.

- Vivienda con cerámica, servicios públicos (electricidad, agua potable, telefonía, servicios de cable) en condiciones de vida óptima. 
2) Categoría U2 (urbana-media):

- Casa de habitación con un nivel medio, con las condiciones básicas para una vida promedio.

- Vivienda con una habitación, servicios básicos.

3) Categoría U3 (urbana-baja):

- Casa de habitación de condiciones mínimas.

- No se consideran costos deacabados con cerámica, vidrio, repello, pulido de paredes y pintura.

- No se consideran costos de excavación en el terreno.

- Se considera solo mobiliario básico, por ejemplo, no cuenta con estufa eléctrica, lavadora de ropa o varios aparatos electrónicos.

b. Zona rural:

1) Categoría Ru1 (rural media):

- Casa de habitación en zona rural bastante parecidas a la categoría U3 (urbana-baja).

2) Categoría Ru2 (rural baja):

- Habitación en condiciones precarias en todos los sentidos humanos y sanitarios posibles.

2. Estimación de daños directos

La estimación del costo de construcción de la vivienda incluye el costo presupuestado de materiales y mano de obra para una vivienda promedio, es decir, de 2 habitaciones, sala, comedor y cocina; además, se considera un porcentaje por la plusvalía de la zona donde está ubicada la misma. A este valor se le sumó el costo del mobiliario básico de una casa, incluyendo solo los electrodomésticos básicos, camas, muebles de sala e implementos de cocina.

a. Para el cálculo de los daños directos en viviendas destruidas y afectadas se tomó en consideración lo siguiente: 
1) Costos de mano de obra para la construcción de una vivienda de $14 \times 25 \mathrm{~m}$, con paredes de bloque.

2) Costode limpieza oremoción de escombros del terreno deconstrucción.

3) Costo de materiales de construcción.

4) Costo de mobiliario promedio de una vivienda para una familia de 5 personas.

b. Para la estimación de daños indirectos se consideró lo siguiente:

1) Recolección de escombros y material por vivienda destruida o dañada.

2) Alquiler de equipo de construcción para la recolección y transporte al lugar de destino.

3) Se calcularon los daños indirectos (pérdidas) correspondientes a la remoción de escombros de viviendas afectadas, en las cuales se tomó como base la cantidad de construcción por $\mathrm{m}^{2}$ en una vivienda estándar; junto con la información brindada por el boletín estadístico de la CHICO: alquiler del equipo, edad de la máquina, lugar del proyecto, tiempo mínimo estimado de trabajo, tiempo de arrendamiento y se estimó el costo por remoción de escombros para viviendas dañadas total 0 parcialmente.

3. Costo de viviendas dañadas o afectadas parcialmente

La estimación monetaria de viviendas afectadas parcialmente se obtuvo a partir de ciertas ponderaciones pertinentes que menciona la Metodología de evaluación de impacto socioeconómico y ambiental de desastres de la CEPAL. Al momento de incluir un costo por daños directos parciales sobre bienes de construcción, inicialmente se estimó como un porcentaje del 30, 40 o 50 por ciento del costo de una vivienda estándar (de acuerdo a la información existente del hecho del desastre vía noticia o también con base a cálculos estadísticos Gumbel para relleno de datos de daños y pérdidas a partir del historial de desastres en la zona y a partir de estimaciones de profesionales de la ingeniería mediante observaciones visuales de la zona). 
Se decidió que para los cálculos de este trabajo, el porcentaje utilizado para poder estimar dichos daños y pérdidas en viviendas dañadas sería de $30 \%$ del costo total de reconstrucción de vivienda, debido a la falta de detalle en las fichas existentes sobre cada uno de los daños en las viviendas y teniendo en cuenta que al momento de estimar los porcentajes de pérdidas por viviendas dañadas parcialmente, entre 40 y 50 porciento los cálculos de costos eran muy altos, según una primera estimación.

Específicamente, para la zona sur del país, se cuenta con fichas en las cuales el número de viviendas afectadas es mayor a 100 viviendas y en algunos casos hasta 600 , en las cuales no se especifica el grado de daño; considerando el elevado número de hogares afectados, se asume que son daños leves o no directos a la infraestructura de las viviendas por tanto se tomó un porcentaje de daño de solo el 5 $\%$.

\section{B. Sector social: salud}

1. Daños directos

Se consideraron como daños directos el costo del tratamiento de un paciente con dengue clásico, que de acuerdo a las autoridades del Hospital Escuela es de siete mil lempiras en promedio y el monto del costo por paciente se incrementa en el caso del dengue hemorrágico, ya que el valor del tratamiento es de entre 15 a 20 mil lempiras.

2. Daños indirectos

Se consideró el costo de distribución del abate en los barrios y colonias, el cual se presupuesta anualmente por las autoridades de Salud y es de entre 19 y 20 millones de lempiras, equivalente a unos 35 mil kilos de abate.

También se asumió el costo de reparaciones y acondicionamiento hecho a la sala de atención de pacientes con dengue del centro hospitalario San Felipe, que fue aprobado por SOPTRAVI, debido a la necesidad de internar a dichos pacientes. La actividad consistió en el cambio de 500 metros cuadrados del techo con estructura metálica, modificaciones en las instalaciones eléctricas, además de otras reparaciones menores en otras salas. El proyecto se llevó a cabo con una inversión de 1.3 millones de lempiras.

a. Sector infraestructura: transporte y comunicaciones (red vial)

Para la estimación de daños y pérdidas se solicitó información acerca de 
daños y pérdidas en materia de infraestructura vial, de las cuales solo fue otorgada información acerca del costo por $\mathrm{m}^{2}$, de reposición de un pavimento asfáltico dado por la oficina de la Dirección General de Carreteras.

También se pidió a la Secretaría de Obras Públicas, Transporte y Vivienda (SOPTRAVI), información referente al número de kilómetros dañados en las diferentes zonas afectadas por desastres. Igualmente, se envió solicitud de información al Fondo Vial referente a proyectos de reconstrucción 0 reparación de tramos carreteros afectados, la cual fue satisfactoriamente aprobada.

Para la estimación de costos en este sector se tomó directamente el costo de reparación de las vías afectadas por lluvias e inundaciones, provista por el Fondo Vial. Algunas de las reparaciones necesarias aún no han sido ejecutadas por falta de fondos, pero se encuentran en su totalidad aprobadas. Cada uno de los proyectos detalla la zona específica en la cual se llevaráa cabo.

b. Sector infraestructura: servicio de distribución de agua potable y saneamiento

EI SANAA proporcionó información sobre los costos de reparación de acueductos y alcantarillados dañados debido a desastres de origen natural durante el 2010, el siguiente paso fue correlacionar los datos con la zona sur del país y en el Municipio del Distrito Central, identificando las fechas y desastres a las cuales correspondían.

Para el Municipio del Distrito Central se identificaron un total de 19 fichas que registran daños en este sector. Además, se encontró que algunas de las reparaciones hechas por el SANAA aún no estaban registradas como daños en el sistema DesInventar, por lo que se procedió a clasificarlas dependiendo de la fecha y zona, para saber a qué desastre natural corresponden y poder ingresarlas en el sistema DesInventar, esto generó un total de 4 fichas, para las cuales se da un monto específico del costo de la reparación.

c. Sector infraestructura: energía

Para la estimación de daños en el sector de energía se solicitó información a la ENEE, dicha institución brindó un cuadro resumen con las reparaciones que se realizaron al sistema de suministro de energía eléctrica, especificando la fecha de la reparación, la ubicación y el monto en lempiras de dicha 
reparación.

Por tanto, se trabajó directamente con los montos provistos por la ENEE, correlacionándolos con las diferentes fichas existentes en el sistema DesInventar.

d. Económico: agricultura y ganadería

En esta sección se consideraron los daños en agricultura y ganadería (cabezas de ganado, cultivos de maicillo, plátano, palma africana, caña de azúcar, frijol, arroz, papa y sorgo, entre otros), que fueron estimados por la SAG para el departamento de Francisco Morazán y El Paraíso, correspondientes a los desastres de origen natural con mayor trascendencia. Los daños directos sobre cultivos se calcularon utilizando el costo de producción de cada uno de los productos o insumos, por quintales o libras.

e. Otros: medioambiente

1) Daños directos en bosques debido a incendios forestales

El Instituto Nacional de Conservación y Desarrollo Forestal, Áreas Protegidas y Vida Silvestre (ICF) se encuentra desarrollando una metodología para el análisis de daños directos e indirectos a bosques, reservas forestales y áreas protegidas, a causa de incendios y plagas; tomando como referencia trabajos realizados anteriormente por estudiantes de la Escuela Agrícola Panamericana El Zamorano y también otra metodología del impacto ambiental desarrollada por investigadores de Suiza, en la cual se evaluó específicamente la biósfera del río Plátano. Los estudiantes de la Escuela Agrícola Panamericana estimaron el monto de L.7,000.00 para daños directos por hectárea de bosque quemada.

Por otra parte, la investigación llevada a cabo en la biósfera del río Plátano que considera la reposición de plantas, la emisión de dióxido de carbono $\left(\mathrm{CO}_{2}\right)$ a la atmósfera, pérdidas de fauna (especies protegidas) y restauración del suelo quemado, entre otros parámetros, estableció un costo de L. 900,000.00 por hectárea quemada de la biósfera. Por lo cual, el ICF, tomando consideraciones de ambas investigaciones previas, ha desarrollado su propia metodología (documento en proceso de publicación) en la cual establece el costo por daños directos como un rango desde los L.15,000.00 considerando bosques con poca infraestructura de vigilancia, poco poblado de flora y fauna; hasta los $L$. $20,000.00$ en donde sí exista infraestructura de vigilancia y mayor población de vida silvestre (ICF, 2011). 
En el caso particular del área de El Picacho, se estableció un monto de L. 22,000.00 debido a la existencia de un zoológico; en consecuencia, considerando la metodología desarrollada por los especialistas nacionales y extranjeros, se decidió considerar el monto promedio de las estimaciones utilizadas por el ICF, es decir, L.17,500.00 por hectárea, como monto por daño directo.

2) Daños indirectos o pérdidas en bosques debido a incendios forestales

Se consideraron en este apartado los costos en los que incurre el ICF, tales como medidas de prevención o mitigación de incendios forestales y plagas, los cuales esta detallados en la tabla 1.

Tabla 1. Medidas de prevención o mitigación ante incendios forestales (variables de ajuste a considerar en el procedimiento de cálculo del precio base en áreas bajo manejo forestal)

\section{Descripción}

Valor en Lps.

Ajuste por establecimiento de viveros forestales: costo por planta lista para plantación.

Ajuste por establecimiento de plantaciones forestales: determina las 8.56 actividades y el costo correspondiente necesarias para establecer apropiadamente una plántula en el sitio definitivo.

Ajuste por construcción de rondas cortafuegos: incluye el costo de construcción por kilómetro de ronda corta fuego con una dimensión de 5 metros de ancho, limpiando con machete y rastrillo-azadón, 1 metro en cada lado de la ronda y quemando el centro.

Ajuste por la realización de quemas prescritas: Las quemas a realizar, se prescriben principalmente para la protección de bosques jóvenes, en regeneración natural, plantaciones establecidas así como otras que se consideren de importancia.

Ajuste por vigilancia en el marco de la protección forestal. Ajuste por combate de incendios forestales: dado que esta actividad corresponde a la protección contra incendios de toda el área bajo manejo (promedio 7,000 has), se plantea como un incentivo de Lps. 20.00 por hectárea no afectada por incendio forestal.

Ajuste por coordinación de regeneración de campaña de protección y asistencia técnica.

Ajuste por manejo de regeneración natural y plantaciones (podas, limpiezas y aclareos).

Ajuste por evaluación de la regeneración natural.

$1,593.75$ por ha

15.13 po ha

20.00

Fuente: elaboración propia con datos del ICF. 


\section{RESULTADOS}

Los resultados de esta investigación se resumen a continuación:

1. Se elaboró un presupuesto de construcción de una casa estándar de área $24 \times 15$ metros para cada una de las categorías definidas, lo cual representa un buen aporte a la metodología que se propone.

2. Se elaboró una metodología para la estimación de daños y pérdidas para las diferentes áreas bajo estudio, considerando su reproducción en otras zonas del país.

3. Se generaron mapas de estimación de daños y pérdidas por desastres para el año 2010 para las zonas de estudio.

4. La tabla 2 resume la estimación de daños y pérdidas para el Distrito Central, para los demás municipios del departamento de Francisco Morazán y para el departamento de El Paraíso, para el sector social: viviendas y asentamientos humanos.

Tabla2. Resumen de estimación de daños y pérdidas sector social: viviendas y asentamientos humanos

\begin{tabular}{|c|c|c|c|c|}
\hline $\begin{array}{l}\text { Sector Social: } \\
\text { Viviendas y }\end{array}$ & Viv. afectadas & $\begin{array}{l}\text { M. Distrito Central } \\
\text { Francisco Morazán } \\
\text { El Paraíso }\end{array}$ & $\begin{array}{l}45,168,775.99 \\
40,849,516.32 \\
52,183,597.75\end{array}$ & $138,201,890.05$ \\
\hline $\begin{array}{l}\text { asentamientos } \\
\text { humanos }\end{array}$ & Viv. destruídas & $\begin{array}{l}\text { Tegucigalpa } \\
\text { Francisco Morazán } \\
\text { El Paraíso }\end{array}$ & $\begin{array}{r}57,203,066.00 \\
29,379,152.67 \\
175,039,728.00\end{array}$ & $261,621,946.67$ \\
\hline
\end{tabular}

*Los valores monetarios están expresados en lempiras.

5. La tabla 3 resume la estimación de daños y pérdidas del sector social: viviendas y asentamientos humanos indicando número de colonias y municipios afectados.

Tabla 3. Resumen de estimación de daños y pérdidas del sector social: viviendas y asentamientos humanos indicando número de colonias y municipios afectados

\begin{tabular}{|l|c|c|c|r|r|r|}
\hline $\begin{array}{c}\text { Zona } \\
\text { geográfica }\end{array}$ & $\begin{array}{c}\text { Número de } \\
\text { municipios }\end{array}$ & $\begin{array}{c}\text { Monto de daños y } \\
\text { colonias } \\
\text { afectadas }\end{array}$ & $\begin{array}{c}\text { Monto de daños } \\
\text { destruidas }\end{array}$ & $\begin{array}{l}\text { Viviendas } \\
\text { afectadas }\end{array}$ & $\begin{array}{c}\text { Mstimación total de } \\
\text { pérdidas por } \\
\text { viviendas destruidas }\end{array}$ & $\begin{array}{c}\text { yérdidas por } \\
\text { viviendas afectadas y pérdidas en } \\
\text { lempiras }\end{array}$ \\
\hline $\begin{array}{l}\text { M. Distrito } \\
\text { Central }\end{array}$ & 99 & 40 & 512 & $12,034,290.02$ & $45,168,775.99$ & $57,203,066.00$ \\
\hline $\begin{array}{l}\text { Francisco } \\
\text { Morazán }\end{array}$ & 13 & 111 & 962 & $29,379,152.67$ & $40,849,516,32$ & $70,228,668.99$ \\
\hline El Paraíso & 6 & 337 & 605 & $175,039,728.00$ & $52,183,597.75$ & $227,223,325.75$ \\
\hline TOTAL & 118 & 488 & 2079 & $216,453,170.69$ & $138,201,890.06$ & $354,655,060.74$ \\
\hline
\end{tabular}

*Los valores monetarios están expresados en lempiras. 
6. Sector social: salud. La tabla 4 resume los daños y pérdidas registrados para el Municipio del Distrito Central, los cuales alcanzan los 369 millones de lempiras. Debe considerarse que el Municipio del Distrito Central alberga dos de los centros hospitalarios más grandes del país, Hospital Escuela y Hospital San Felipe, que prestan sus servicios a un amplio rango de población de las áreas rurales, por lo cual es justificable el elevado número de atenciones médicas de pacientes con dengue clásico como hemorrágico.

7. La tabla 4 muestra la estimación de daños y pérdidas durante el año 2010 para el sector social: salud para el Municipio del Distrito Central, los demás municipios del departamento de Francisco Morazán y el departamento de El Paraíso.

Tabla 4. Estimación de daños y pérdidas durante el año 2010, sector social: salud

\begin{tabular}{|c|c|c|c|c|}
\hline \multicolumn{5}{|c|}{ Municipio del Distrito Central } \\
\hline Daños & Descripción & $\begin{array}{l}\text { Número de } \\
\text { pacientes }\end{array}$ & $\begin{array}{l}\text { Estimación de } \\
\text { costos (lempiras) }\end{array}$ & $\begin{array}{l}\text { Estimación de } \\
\text { costos ( } \$ \text { US) }\end{array}$ \\
\hline \multirow{2}{*}{ Directos } & $\begin{array}{l}\text { Atención de pacientes con } \\
\text { dengue clásico }\end{array}$ & $33,066.00$ & $231,462,000.00$ & $245,370,86$ \\
\hline & $\begin{array}{l}\text { Atención de pacientes con } \\
\text { dengue hemorrágico }\end{array}$ & $7,834.00$ & $137,095,000.00$ & $7,252,936.20$ \\
\hline \multirow[t]{3}{*}{ Indirectos } & $\begin{array}{l}\text { Acondicionamiento sala } \\
\text { de atención Hospital San } \\
\text { Felipe }\end{array}$ & - & $1,300.000 .00$ & $68,775.79$ \\
\hline & $\begin{array}{l}\text { Acciones de control y } \\
\text { operativos de salud }\end{array}$ & - & $30,092.59$ & $1,592.03$ \\
\hline & TOTAL & $40,900.00$ & $369,887,092.59$ & $19,568,674.88$ \\
\hline \multicolumn{5}{|c|}{ Francisco Morazán } \\
\hline Daños & Descripción & $\begin{array}{l}\text { Número de } \\
\text { pacientes }\end{array}$ & $\begin{array}{l}\text { Estimación de } \\
\text { costos (lempiras) }\end{array}$ & $\begin{array}{l}\text { Estimación de } \\
\text { costos (\$ US) }\end{array}$ \\
\hline \multirow{2}{*}{ Directos } & $\begin{array}{l}\text { Atención de pacientes con } \\
\text { dengue clásico }\end{array}$ & $36,342.00$ & $254,394,000.00$ & $13,375,078.86$ \\
\hline & $\begin{array}{l}\text { Atención de pacientes con } \\
\text { dengue hemorrágico }\end{array}$ & $8,016.00$ & $140,280,000.00$ & $7,375,394.32$ \\
\hline \multirow[t]{2}{*}{ Indirectos } & $\begin{array}{l}\text { Acciones de control y } \\
\text { operativos de salud }\end{array}$ & - & $812,500.00$ & $42,718.19$ \\
\hline & TOTAL & $44,358.00$ & $395,486,500.00$ & $20,793,191.38$ \\
\hline \multicolumn{5}{|c|}{ El Paraíso } \\
\hline Daños & Descripción & $\begin{array}{l}\text { Número de } \\
\text { pacientes }\end{array}$ & $\begin{array}{c}\text { Estimación de } \\
\text { costos (lempiras) }\end{array}$ & $\begin{array}{l}\text { Estimación de } \\
\text { costos ( } \$ \text { US) }\end{array}$ \\
\hline \multirow{2}{*}{ Directos } & $\begin{array}{l}\text { Atención de pacientes con } \\
\text { dengue clásico }\end{array}$ & $4,654.00$ & $32,578,000.00$ & $1,712,828.60$ \\
\hline & $\begin{array}{l}\text { Atención de pacientes con } \\
\text { dengue hemorrágico }\end{array}$ & $2,046.00$ & $35,805,000.00$ & $1,882,492.11$ \\
\hline \multirow[t]{2}{*}{ Indirectos } & $\begin{array}{l}\text { Acciones de control y } \\
\text { operativos de salud }\end{array}$ & - & $541,666.67$ & $28,478.79$ \\
\hline & TOTAL & $6,700.00$ & $68,924,666.67$ & $3,523,799.51$ \\
\hline
\end{tabular}


8. Sector infraestructura: distribución de agua potable y saneamiento. Los costos de reparación de acueductos y alcantarillados en los diferentes barrios y colonias de las zonas de estudio se detallan en la tabla 5 para el Municipio del Distrito Central y los departamentos de Francisco Morazán y El Paraíso.

9. El Distrito Central muestra un total de 19 fichas en las cuales se registran daños, mientras que el departamento de Francisco Morazán muestra 2 fichas y el departamento de El Paraíso muestra un total de 20 fichas.

10. Para el Municipio del Distrito Central se identificaron 14 fichas como reparaciones comunes, que hacienden a un monto de L. 5,357.14 por ficha, lo cual implica un monto de L. $75,000.00$. Las restantes 5 fichas detallan proyectos específicos de mayor magnitud, que juntas ascienden a un monto de 2.32 millones de lempiras; de esta forma, el impacto total para el Municipio del Distrito Central es de 2.4 millones de lempiras. En el caso del departamento de Francisco Morazán, se registran daños por 3.8 millones de lempiras distribuidos en 2 fichas y para el departamento de El Paraíso se registraron 20 fichas, con un monto total de daños de 4.8 millones de lempiras.

Tabla 5. Estimación de daños y pérdidas para el Municipio del Distrito Central y demás municipios de Francisco Morazán y El Paraíso para el sector infraestructura: distribución de agua potable y saneamiento

\begin{tabular}{l|c|r|r|}
\hline \multirow{2}{*}{ Zona } & \multirow{2}{*}{ No. de fichas } & \multicolumn{2}{c|}{ Estimación de daños y pérdidas } \\
\cline { 3 - 4 } & 19 & Total (lempiras) & \multicolumn{1}{c|}{ Total (US dólares) } \\
\hline $\begin{array}{l}\text { M. Distrito Central } \\
\text { Francisco }\end{array}$ & 2 & $2,401,959.96$ & $126,286.01$ \\
\hline $\begin{array}{l}\text { Morazán } \\
\text { El Paraíso }\end{array}$ & 20 & $4,811,448.00$ & $200,391.59$ \\
\hline \multicolumn{1}{|c|}{ TOTAL } & & $4,864,000.00$ & $255,730.81$ \\
\hline
\end{tabular}

11. Sector infraestructura: energía. La tabla 6 detalla los costos de reparación de líneas de tendido eléctrico en el Municipio del Distrito Central durante el año 2010; son un total de 56 fichas que representan un monto de 281 mil lempiras. No se lograron obtener datos para los departamentos de Francisco Morazán y El Paraíso.

Tabla 6. Estimación de daños y pérdidas para el Municipio del Distrito Central para el sector infraestructura: energía

\begin{tabular}{|c|c|r|r|}
\hline \multirow{2}{*}{ Zona } & \multirow{2}{*}{ No. de fichas } & \multicolumn{2}{|c|}{ Estimación de daños y pérdidas } \\
\cline { 3 - 4 } & & Total (lempiras) & \multicolumn{1}{c|}{ Total (US dólares) } \\
\hline M. Distrito Central & 56 & $2,891,723.02$ & $14,905.82$ \\
\hline
\end{tabular}


12. Sector infraestructura: transporte y comunicaciones. Los costos debido a reparaciones en los diferentes tramos carreteros afectados por desastres de origen natural están detallados en la tabla 7 para el Municipio del Distrito Central; tal como se observa, se registran daños por 53 millones de lempiras. No se lograron obtener datos para los demás municipios de los departamentos de Francisco Morazán y ElParaíso.

Tabla 7. Estimación de daños y pérdidas para el Municipio del Distrito Central para el sector infraestructura: transporte y comunicaciones

\begin{tabular}{|c|c|c|c|}
\hline \multirow{2}{*}{ Zona } & \multirow{2}{*}{ No. de fichas } & \multicolumn{2}{|c|}{ Estimación de daños y pérdidas } \\
\cline { 3 - 4 } & & Total (lempiras) & \multicolumn{1}{c|}{ Total (US dólares) } \\
\hline M. Distrito Central & 56 & $53,075,119.47$ & $366,166.43$ \\
\hline
\end{tabular}

13. Sector económico: agricultura y ganadería. La tabla 8 registra los daños en el sector económico: agricultura y ganadería, para los departamentos de Francisco Morazán y EI Paraíso. La estimación monetaria de daños y pérdidas realizada por la SAG asciende a 5,706,046.42 lempiras en materia de cultivos y ganado para Francisco Morazán y EI Paraíso, pero debido a la falta de información específica de cada sector, la misma se distribuyó equitativamente entre las diferentes fichas en las cuales coincidían las fechas con el evento natural registrado como causante de los daños y pérdidas.

Tabla 8. Estimación de daños y pérdidas para los departamentos de Francisco Morazán y El Paraíso para el sector económico: agricultura y ganadería

\begin{tabular}{|l|r|r|r|}
\hline \multicolumn{1}{|c|}{ Zona } & $\begin{array}{c}\text { Número de hectáreas } \\
\text { afectadas }\end{array}$ & \multicolumn{1}{c|}{ Costo total Lps. } & Costo total. dolares (US) \\
\hline Francisco Morazán & $1,640.00$ & $70,484,708.00$ & $3,705,820.61$ \\
\hline EI Paraíso & $6,687.00$ & $38,044,295.00$ & $2,000,225.81$ \\
\hline TOTAL & $8,327.00$ & $108,529,003.00$ & $5,706,046.42$ \\
\hline
\end{tabular}

14. Otros sectores: medioambiente. La tabla 9 muestra la estimación de daños y pérdidas para el sector de medioambiente para el Municipio del Distrito Central, los demás municipios de los departamentos de Francisco Morazán y El Paraíso. 
Tabla 9. Estimación de daños y pérdidas para los departamentos de Francisco Morazán y El Paraíso para el sector medioambiente

\begin{tabular}{|l|r|r|r|}
\hline \multicolumn{1}{|c|}{ Zona } & $\begin{array}{c}\text { Número de hectáreas } \\
\text { quemadas }\end{array}$ & Costo total Lps. & Costo total. dolares (US) \\
\hline M. Distrito Central & 490.88 & $22,238,650.00$ & $1,169,224.54$ \\
\hline Francisco Morazán & $1,525.15$ & $69,094.846 .55$ & $3,632,746.93$ \\
\hline El Paraíso & $5,659.38$ & $256,390,514.14$ & $13,480,048.06$ \\
\hline TOTAL & $7,675.41$ & $347,724,011.49$ & $18,282,019.53$ \\
\hline
\end{tabular}

15. La tabla 10 presenta el resumen de la estimación de daños y pérdidas para los diferentes sectores analizados en las zonas de estudio.

Tabla 10. Resumen de estimación de daños y costos para los diferentes sectores analizados

\begin{tabular}{|c|c|c|c|c|c|c|c|c|}
\hline & & \multicolumn{7}{|c|}{ Estimado de daños y pérdidas } \\
\hline Sector & & $\begin{array}{c}\text { Municipio del } \\
\text { Distrito Central } \\
\text { (lempiras) }\end{array}$ & $\begin{array}{l}\text { Francisco } \\
\text { Morazán } \\
\text { (lempiras) }\end{array}$ & $\begin{array}{l}\text { El Paraíso } \\
\text { (lempiras) }\end{array}$ & $\begin{array}{c}\text { Total } \\
\text { lempiras }\end{array}$ & $\begin{array}{l}\text { Total } \\
\text { dolares } \\
\text { (US) }\end{array}$ & $\begin{array}{l}\text { Total por } \\
\text { sector } \\
\text { lempiras }\end{array}$ & $\begin{array}{l}\text { Total por } \\
\text { sector } \\
\text { dolares (US) }\end{array}$ \\
\hline \multirow[t]{2}{*}{ Social } & $\begin{array}{l}\text { Vivienda y } \\
\text { asentamientos } \\
\text { humanos }\end{array}$ & $57,203,066.00$ & $117,273,114.49$ & $227,223,325.75$ & $401,699,506.24$ & $21,119,847.86$ & \multirow[t]{2}{*}{$1,235,997,765.50$} & \multirow[t]{2}{*}{$64,984,109.65$} \\
\hline & Salud & $369,887,092.59$ & $395,486,500.00$ & $68,924,666.67$ & $834,924,666.67$ & $43,864,261.79$ & & \\
\hline \multirow[t]{2}{*}{ Infraestructura } & $\begin{array}{l}\text { Transporte y } \\
\text { Comunicaciones } \\
\text { Distribución de } \\
\text { agua potable y }\end{array}$ & $\begin{array}{r}53,075,119.47 \\
2,401,959.96 \\
\end{array}$ & $3,811,448.00$ & $4,864,000.00$ & $\begin{array}{r}53,075,119.47 \\
11,077,407.96\end{array}$ & $\begin{array}{r}2,790,489.98 \\
582,408.41\end{array}$ & \multirow[t]{2}{*}{$64,434,250.45$} & \multirow[t]{2}{*}{$3,387,710.33$} \\
\hline & $\begin{array}{l}\text { saneamiento } \\
\text { Energía }\end{array}$ & & & & 281.723 .02 & & & \\
\hline Económico & $\begin{array}{l}\text { Agricultura y } \\
\text { ganadería }\end{array}$ & $\begin{array}{r}0.02 \\
-\end{array}$ & $70,484,708.00$ & $38,044,295.00$ & $108,529,003.00$ & $\begin{array}{r}14,811.94 \\
5,706,046,42\end{array}$ & $108,529,003.00$ & $5,706,046.42$ \\
\hline Otros & Medio Ambiente & $22,238,650.80$ & $69,094,846.55$ & $256,390,514.14$ & $347,724,011.49$ & $18,282,019.53$ & $347,724,011.49$ & $18,282,019.53$ \\
\hline
\end{tabular}

16. El mapa 2 presenta la estimación del costo por daños y pérdidas para la zona de estudio para el sector social: viviendas y asentamientos humados. 
Mapa 2. Estimación del costo por daños y pérdidas sector social: viviendas y asentamientos humanos

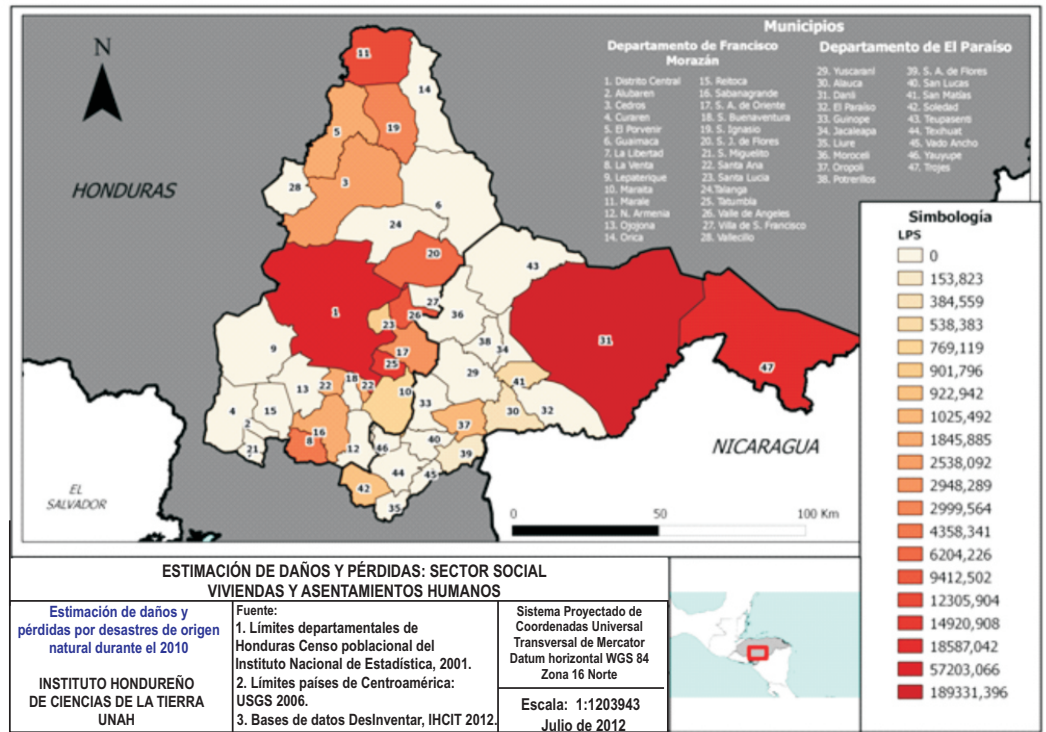

17. El mapa 3 presenta la estimación del costo por daños y pérdidas para la zona de estudio para el sector social: salud.

Mapa 3. Estimación del costo por daños y pérdidas sector social: salud

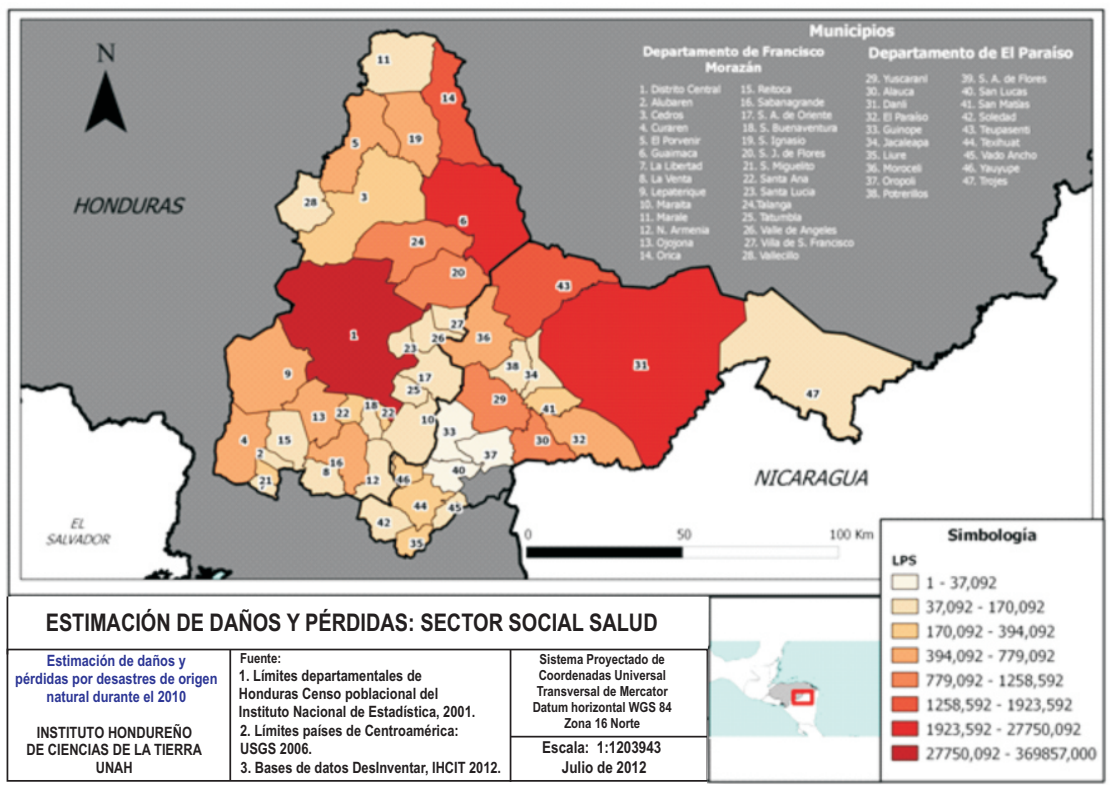


18. El mapa 4 presenta la estimación del costo por daños y pérdidas para la zona de estudio para el sector económico: agricultura y ganadería.

Mapa4. Estimación del costo por dañosy pérdidas sector social: agricultura y ganadería

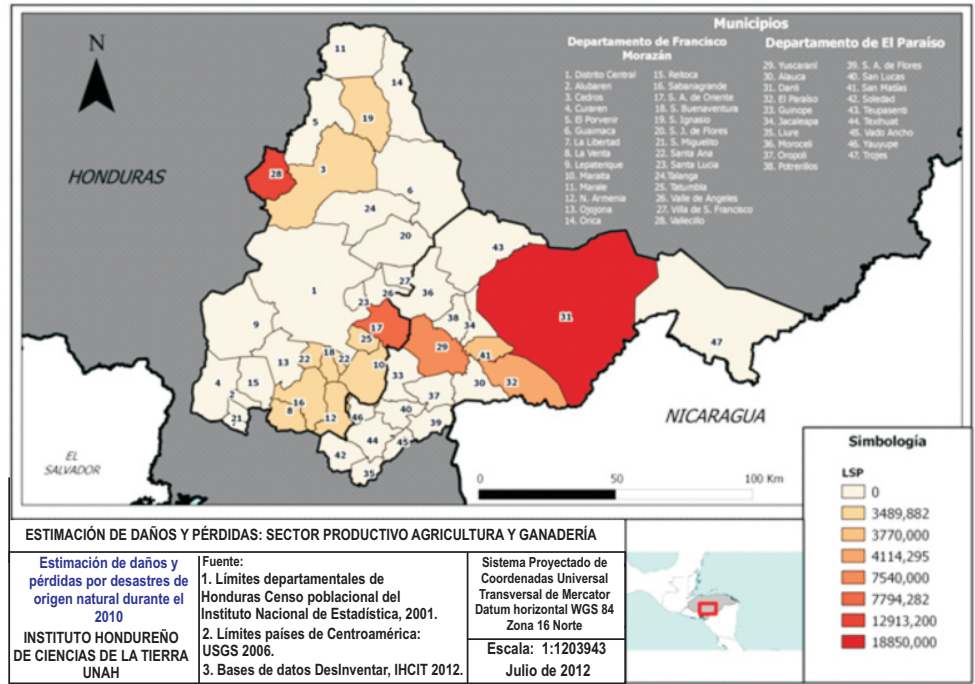

19. El mapa 5 presenta la estimación del costo por daños y pérdidas para la zona de estudio para el sector medioambiente.

Mapa 5. Estimación del costo por daños y pérdidas sector medioambiente

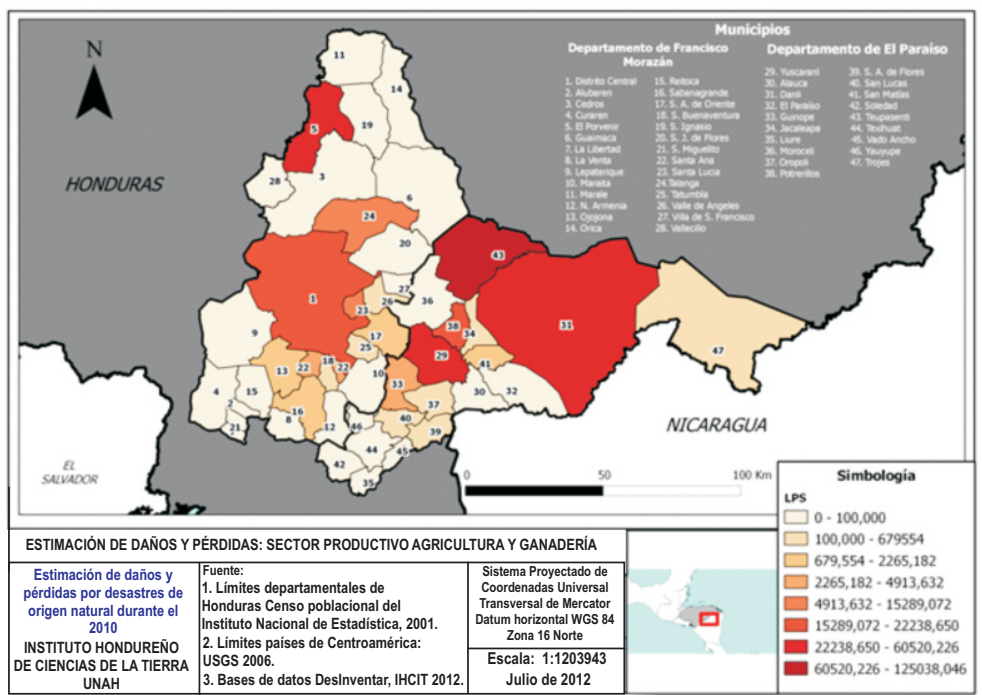




\section{DISCUSIÓN DE RESULTADOS}

El resultado de la estimación de daños y pérdidas en el sector social: viviendas y asentamientos humanos, es uno de los más altos obtenidos durante el estudio para la zona seleccionada, representando un $29.7 \%$.

Los eventos de mayor frecuencia e impacto durante el año 2010, para las zonas bajo estudio, fueron las inundaciones y deslizamientos causados por lluvias.

Un factor que interviene en el impacto de los daños y pérdidas es la existencia de muchas familias que habitan en zonas de alto riesgo; así, las llamadas invasiones de barrios y colonias son muy comunes en la periferia de la capital hondureña, por ejemplo, donde se hacen construcciones sin tomar en cuenta las medidas de seguridad y en muchos casos son viviendas precarias, en terrenos propensos a deslizamiento 0 inundaciones. Es sabido que el aspecto económico tiene mucha influencia en estas actividades, pero también debe considerarse el papel que deben desempeñar las autoridades municipales al permitir este tipo de procesos.

La estimación de daños y pérdidas para el sector social salud, para toda la zona de estudio, dio como resultado la alarmante suma de 22.6 millones de dólares (430 millones de lempiras), siendo este subsector el más alto de todos los estudiados, lo cual implica que la salud es uno de los subsectores más frágiles para la población hondureña. Analizando la estimación de daños en este sector, se puede observar que del total estimado, el $99.7 \%$ (es decir 428 millones de lempiras) se invirtieron en la atención de emergencia a la epidemia del dengue y solo un 0.03 \% (1.47 millones de lempiras) se invirtieron en las acciones de mitigación y prevención de la misma, lo cual señala una endeble preparación ante una amenaza que persiste año tras año para la población hondureña. Un aumento del presupuesto para prevención de una nueva epidemia de dengue, generaría una reducción significativa para el estado del gasto por atención de pacientes en hospitales públicos.

El sector infraestructura transporte y comunicaciones presenta daños y pérdidas por el orden de 53 millones de lempiras, siendo uno de los sectores que es fuertemente afectado por desastres de origen natural y debido al enorme impacto que tiene en la economía de la nación como un todo, considerando que daños en tramos carreteros dejan incomunicados a sectores de la población durante días o semanas, o en otros casos obligados a usar vías altamente congestionadas durante largos periodos de tiempo, como sucede con el tráfico del bulevar Fuerzas Armadas del Municipio del Distrito Central, que debido a los efectos de la tormenta Agatha colapsó en uno de sus tramos, quedando así el flujo de vehículos de la parte norte de la ciudad 
imposibilitado por esta vía durante cerca de 6 meses. Esto generó enormes implicaciones en el tráfico en otras vías de la ciudad capital, provocando pérdida de tiempo y dinero tanto para la población, como para la empresa privada que utilizaba esta vía para el transporte interurbano de productos e insumos. Por tanto, es innegable el efecto dominó que ejerce sobre la economía los daños en el sector infraestructura transporte y comunicaciones.

Al considerar el sector infraestructura distribución de agua potable y saneamiento, se observó que este sector representa $0.82 \%$ del total de daños y pérdidas estimados, en consecuencia, debe considerarse que los daños en este sector afectan el acceso al agua potable para la población, el cual es una necesidad básica, de manera que la falta de prevención de estos efectos representa una fragilidad para la salud de la población.

En el sector económico agricultura y ganadería se registran daños y pérdidas por 108.5 millones de lempiras (5.7 millones de dólares) y considerando que este es uno de los sectores más importantes por su relevancia no solo a nivel monetario, sino en la seguridad alimentaria de los ciudadanos, el impacto puede generar un efecto dominó sobre la economía del resto de la población, logrando de esta manera que la totalidad de la población hondureña sufra los efectos de los desastres de origen natural en forma de un incremento a los precios de los productos alimenticios que son indispensables (canasta básica) o falta de abastecimiento de determinados productos nacionales como el maíz, frijol, arroz, productos lácteos y derivados, entre otros.

Estos efectos fueron ocasionados en su mayoría por eventos del tipo inundaciones, debido a lluvias excesivas, ya que a partir del año 2010 de sintió la presencia del fenómeno de La Niña (en 2009 fue El Niño), lo cual implicó un aumento en el promedio de las precipitaciones, eventos para los cuales la población no estaba preparada.

El sector energía, con un porcentaje de $0.02 \%$, muestra la fragilidad a la que están expuestos ciertos sectores para los cuales es indispensable el servicio de energía eléctrica, tales como hospitales e industria. Estos daños se dieron en la temporada lluviosa, normalmente provocados por tormentas eléctricas que dañaron esporádicamente algunos postes, cableado eléctrico y transformadores, principalmente en la zona de Tegucigalpa.

Se observó el sector medioambiente con un porcentaje del $25.71 \%$, del total de daños y pérdidas en el año 2010. Es importante destacar que los efectos de los 
incendios forestales son significativos y dañinos para el medioambiente a mediano plazo y este tipo de daños no son representables en su totalidad como una suma monetaria y son difíciles de revertir. Estos eventos también generan daños indirectos a la salud de la población a largo plazo, en forma de enfermedades respiratorias.

La información sobre cada uno de los eventos y sectores analizados en este estudio, es de mucha importancia para las autoridades municipales y gubernamentales, ya que permitirá la toma de decisiones adecuadas enfocadas en la prevención y mitigación de desastres. Por tanto, el sistema DesInventar cumple esta importante misión de registrar dichos daños para su posterior análisis.

\section{AGRADECIMIENTOS}

Se agradece a la Dirección de Investigación Científica (DICU) de la Universidad Nacional Autónoma de Honduras, ya que sin su apoyo financiero este proyecto no hubiese sido posible.

Gracias a Angie Murillo por su asesoría con relación a la metodología de la CEPAL y a Nabil Kawas, director del Instituto Hondureño de Ciencias de la Tierra (IHCIT) por el apoyo brindado al proyecto.

Igualmente gracias a todas las instituciones que colaboraron con esta investigación: Instituto de Conservación y Desarrollo Forestal y Vida Silvestre (ICF), Empresa Nacional de Energía Eléctrica (ENEE), Secretaría de Agricultura y Ganadería (SAG), Servicio Autónomo Nacional de Acueductos y Alcantarillados (SANAA), Fondo Vial y Consultores de Ingeniería (CINSA) y asociados.

\section{BIBLIOGRAFÍA}

Cámara Hondureña de la Industria de la Construcción (2010). Expo-construyendo 2010. Boletín estadístico.

Comisión Económica para América Latina. (2003). Metodología de evaluación del impacto socioeconómico y ambiental de desastres.

Empresa Nacional de Energía Eléctrica. (2010). Información referente a reparaciones al sistema de suministro de energía eléctrica, dañadas debido a vientos muy potentes y lluvias excesivas, realizadas durante el año.

Fondo Vial. (2011). Cuadro resumen sobre reparaciones a tramos carreteros afectados por desastres de origen natural. 
IHCIT-UNAH. (2012). Sistema de inventario de desastres de origen natural Desinventar. Disponible en: www.desinventar.org.

Instituto Nacional de Conservación y Desarrollo Forestal, Áreas Protegidas y Vida Silvestre (ICF). Manual de procedimiento de ajuste a la valoración del bosque por concepto de inversiones de manejo forestal en áreas de bosque nacional cedidas bajo contrato.

Secretaría de Agricultura y Ganadería (2011). Estadísticas de daños y pérdidas en ganadería y por cultivos de maíz, frijol y sorgo entre otros.

Servicio Nacional de Acueductos y Alcantarillado. Descripción de costos por reparación de acueductos y alcantarillados dañados por desastres de origen natural durante el año 2010. 